Ciência Florestal, Santa Maria, v. 26, n. 1, p. 1-9, jan.-mar., 2016

ISSN 0103-9954

\title{
CRESCIMENTO INICIAL DE ACÁCIA-NEGRA COM VERMICOMPOSTOS DE DIFERENTES RESÍDUOS AGROINDUSTRIAIS
}

\author{
INITIAL GROWTH OF Acacia mearnsii WITH VERMICOMPOST OF DIFFERENT \\ AGROINDUSTRIAL WASTES
}

\author{
Rul Martins Antunes ${ }^{1}$ Rosa Maria Vargas Castilhos ${ }^{2}$ Danilo Dufch Castilho ${ }^{3}$ \\ Otávio dos Anjos Leal ${ }^{4}$ Robson Andreazza ${ }^{5}$
}

\begin{abstract}
RESUMO
A utilização de vermicompostos de diferentes resíduos agroindustriais na produção de mudas de acácianegra pode ser uma alternativa de reutilização de resíduos e aumentar a produção de mudas. Assim, os objetivos deste trabalho foram avaliar o crescimento e a concentração de nutrientes em mudas de acácianegra, cultivadas em substratos com diferentes vermicompostos de resíduos orgânicos agroindustriais. Instalou-se em casa de vegetação 11 diferentes tratamentos: T1) vermicomposto de esterco bovino (EB); T2) vermicomposto de esterco ovino (EO); T3) vermicomposto de lodo de parbolização de arroz (LP); T4) tratamento controle (sem adubação); T5) tratamento controle com adução mineral (NPK); T6) mistura de EB e LP; T7) mistura de EO e LP; T8) mistura de EB e vermicomposto de resíduos de alimentos (RA); T9) mistura de EO e RA; T10) mistura de EB e vermicomposto de resíduos de frutas (RF); T11) mistura de EO e RF. Após 180 dias de cultivo em recipiente com capacidade de cinco litros, foram analisadas a massa seca e a concentração de nutrientes na parte aérea da acácia-negra, e a concentração de nutrientes no solo, após o cultivo. A adição do esterco bovino, bem como a mistura de esterco bovino e resíduos alimentícios favoreceram o incremento de matéria seca das plantas de acácia-negra. Os resultados mostraram que as concentrações de nutrientes nas plantas, com exceção de Fe e Mn, variaram com adição de vermicompostos no solo. Os tratamentos T3 e T6 elevaram as concentrações em P, N, Zn de Cu nas folhas de acácia-negra. Além disso, a adição dos vermicompostos ao solo aumentou a disponibilidade de nutrientes para as plantas, mesmo após o cultivo, especialmente com relação ao fósforo, potássio e magnésio, sendo uma alternativa viável e eficaz na produção de mudas, podendo substituir a utilização de adubação mineral.
\end{abstract}

Palavras-chave: substratos orgânicos; composto orgânico; nutrição de plantas.

\begin{abstract}
The use of vermicompost as organic compounds of different agro-industrial wastes in the production of Acacia mearnsii seedlings can be an alternative of reusing waste and increase of seedlings production. The aim of this study was to evaluate the growth and nutrient concentration in Acacia mearnsii seedlings grown in different soils and vermicomposts of different organic wastes. Also, the effects on soil chemical properties were evaluated. So, different treatments were applied: T1) vermicompost of bovine manure (EB); T2)

1 Químico, Dr., Professor do Centro de Engenharias, Universidade Federal de Pelotas, Rua Almirante Barroso 1734, Bairro Centro, CEP 96010-280, Pelotas (RS), Brasil. rul1976@gmail.com

2 Engenheira Agrônoma, Dr ${ }^{\mathrm{a}}$., Professora Associada do Departamento de Solos, Faculdade de Agronomia Eliseu Maciel, Universidade Federal de Pelotas, Caixa Postal 354, CEP 96010-900, Pelotas (RS), Brasil. rosamvc@ufpel.tche.br

3 Engenheiro Agrônomo, Dr., Professor Associado do Departamento de Solos, Faculdade de Agronomia Eliseu Maciel, Universidade Federal de Pelotas, Caixa Postal 354, CEP 96010-900, Pelotas (RS), Brasil.

4 Engenheiro Agrônomo, Doutorando em Ciência do Solo da UFRGS - Porto Alegre (RS), Brasil, oleal@ibest.com.br

5 Engenheiro Agrônomo, Dr., Professor do Centro de Engenharias, Universidade Federal de Pelotas, Rua Benjamin Constant 897, CEP 96010020, Pelotas (RS), Brasil. robsonandreazza@yahoo.com.br
\end{abstract}

Recebido para publicação em 31/08/2012 e aceito em 19/05/2014

Ci. Fl., v. 26, n. 1, jan.-mar., 2016 
vermicompost of ovine manure (EO); T3) vermicompost of rice parboiled waste (LP); T4) Control (without amendment); T5) Control with mineral amendment; T6) mixture of EB and LP; T7) mixture of EO and LP; T8) mixture of EB and vermicompost of food wastes (RA); T9) mixture of EO and RA; T10) mixture of EB and vermicompost of fruits wastes (RF); T11) mixture of EO and RF. After 180 days of growth, it was analyzed the dry mass and nutrient concentration in shoots and the concentration of nutrients in the soil after cultivation. The addition of EB, as well as the mixture of EB and RA promoted the increase on dry matter. The results showed that the concentrations of nutrients in plants, with the exception of Fe, Mn varied with the addition of vermicompost in the soil. Treatments $\mathrm{T} 3$ and $\mathrm{T} 6$ increased the concentrations of $\mathrm{P}, \mathrm{N}, \mathrm{Zn}, \mathrm{Cu}$ in leaves of Acacia mearnsii. Furthermore, the addition of vermicompost to soil increased the availability of nutrients to plants, even after cultivation, especially the phosphorus, potassium and magnesium, and it is a viable and effective in producing Acacia mearnsii seedlings and might replace the use of mineral fertilizers. Keywords: agroindustrial wastes; organic compost; plant's nutrition.

\section{INTRODUÇÃO}

A acácia-negra (Acacia mearnsii De Wild.), espécie originada da Austrália, é uma leguminosa arbórea de múltiplos usos, tais como: a restauração de solos de baixa fertilidade ou degradados; produção de tanino, a partir da casca, para uso industrial; produção de madeira para obtenção de energia e/ ou fabricação de celulose e papel (TONETTO e STEIN, 1997). A acácia-negra não tolera solos mal drenados e apresenta desenvolvimento reduzido em solos muito ácidos e de baixa fertilidade natural (HIGA e RESENDE, 1994).

O vermicomposto é um produto orgânico transformado e estabilizado por minhocas, rico em ácidos húmicos (ANTUNES, 2009). A vermicompostagem pode ser realizada com vários substratos, contudo, as minhocas devem ter a aptidão de ingestão e metabolização dos mesmos (ANTONIOLLI et al., 2002). Diferentes tipos de resíduos são utilizados para a vermicompostagem, como esterco bovino, esterco ovino, lodo originado da parbolização do arroz, resíduos de alimentos, resíduos de frutas e legumes (ANTUNES, 2009), lodo de esgoto(SILVAet al., 2010; HASHEMIMAJD e JAMAATI-SOMARIN, 2011), entre outros.

O aproveitamento dos resíduos gerados por diferentes atividades agroindustriais pode ser uma alternativa para redução dos custos com adubação em cultivos florestais. Além disto, podese evitar a contaminação do solo, do ar e da água pela simples deposição dos resíduos, e contribuir de forma significativa para a utilização sustentável dos recursos naturais, melhorando as condições do solo e aumentando o seu nível de fertilidade (TEDESCO et al., 1999). No entanto, alguns resíduos necessitam sofrer decomposição parcial, antes de serem adicionados ao solo, para que não gerem efeitos adversos. Uma das alternativas para o processamento de resíduos orgânicos é a vermicompostagem, processo que utiliza minhocas com agentes decompositores, o mesmo requer pouco consumo de energia e fornece material estabilizado em tempo menor, se comparado com a compostagem (TIBAU, 1984).

A acácia-negra é uma cultura em que promove condições biológicas no solo como as micorrizas (SILVA et al., 2008) e a fixação biológica de nitrogênio através do rizóbio (VARGAS et al., 2007). Além disso, sabe-se que na utilização de resíduos orgânicos, contendo ácidos orgânicos, há a promoção do crescimento de algumas plantas, como por exemplo, o abacaxi, pois proporciona o melhor crescimento e a adaptação de mudas de abacaxizeiro ao ambiente (BALDOTTO et al., 2010). Embora haja alguns trabalhos sobre a produção de mudas de acácia-negra com diferentes substratos (MARTINS et al., 2012) e utilizadas em diferentes sistemas agroflorestais (KLEINPAUL et al., 2010; VIERA et al., 2011) fonte de nutrientes (CALDEIRA et al., 2002; 2004), ainda necessita-se avaliar o efeito da utilização de vermicomposto no crescimento inicial das plantas. Assim, o objetivo deste trabalho foi avaliar o crescimento inicial e a concentração de nutrientes em mudas de acácia-negra, cultivadas em solos adubados com vermicompostos de resíduos orgânicos agroindustriais, bem como o efeito sobre os atributos químicos do solo.

\section{MATERIAL E MÉTODOS}

\section{Caracterização do local e solo}

O experimento foi realizado em casa de 
vegetação e em laboratórios do Departamento de Solos da Faculdade de Agronomia da Universidade Federal de Pelotas. O solo utilizado foi identificado como Argissolo Vermelho Amarelo (STRECK et al., 2002) coletado na camada de 0-20 cm de profundidade. O solo foi caracterizado quimicamente de acordo com a metodologia descrita por Tedesco et al. (1995). As características físico-químicas são as seguintes: $\mathrm{pH}_{\mathrm{H} 2 \mathrm{O}}=4,7 ; \mathrm{P}=3,7 \mathrm{mg} \mathrm{dm}^{-3} ; \mathrm{K}=30$ mg dm${ }^{-3}$; matéria orgânica $=12 \mathrm{~g} \mathrm{~kg}^{-1}$; argila $=230$ $\mathrm{g} \mathrm{kg}^{-1} ; \mathrm{Ca}=0,6 \mathrm{cmol}_{\mathrm{c}} \mathrm{dm}^{-3} ; \mathrm{e} \mathrm{Mg}=0,4 \mathrm{cmol}_{\mathrm{c}} \mathrm{dm}^{-3}$. Com base na análise de solo, foi realizada a calagem para elevar o $\mathrm{pH}$ a 5,5; aplicando-se o equivalente de 1,0 $\mathrm{Mg} \mathrm{ha}^{-1}$ de calcário, de acordo com CQFS (2004).

\section{Tratamentos utilizados}

Foram aplicados onze diferentes tratamentos: com nove vermicompostos, como fonte de fertilizante orgânico; um tratamento controle positivo, com adubação mineral de NPK e um tratamento sem adubação como controle negativo (Tabela 1). Estes foram dispostos inteiramente ao acaso, com três repetições e as unidades experimentais consistiram de vasos plásticos, contendo $5 \mathrm{~kg}$ de solo.

A adubação mineral baseou-se na interpretação da análise de solo e recomendação de adubação, conforme o Manual de Adubação e Calagem para RS e SC (CQFS, 2004), no qual são preconizados para a acácia-negra $45 \mathrm{~kg} \mathrm{ha}^{-1}$ de $\mathrm{N}$, $120 \mathrm{~kg} \mathrm{ha}^{-1}$ de $\mathrm{P}_{2} \mathrm{O}_{5}$ e $70 \mathrm{~kg} \mathrm{ha}^{-1}$ de $\mathrm{K}_{2} \mathrm{O}$. As fontes minerais NPK utilizadas foram: ureia, superfosfato triplo e cloreto de potássio, respectivamente.
As doses dos vermicompostos foram calculadas com base na sua composição química (Tabela 1) e objetivando atender à mesma recomendação de nitrogênio da cultura, em todos os tratamentos com adubação. A Tabela 1 mostra a descrição dos tratamentos, a concentração de nutrientes nos vermicompostos e as quantidades aplicadas.

\section{Cultivo e avaliações}

As sementes de acácia-negra (Acacia mearnsii) foram concedidas pela EMBRAPA, sendo estas sementes germinadas em tubetes laminados com capacidade para $60 \mathrm{~cm}^{3}$ de substrato, este com nome comercial Plantmax (Fabricado pela Buschle \& Lepper S.A.). Após 60 dias da germinação, as mudas uniformes foram transplantadas (uma planta por vaso) e cultivadas por 120 dias sob efeito dos diferentes tratamentos. Diariamente foi realizada a reposição da água evapotranspirada, com água destilada, esta reposição foi realizada por diferença de peso dos vasos, mantendo todos os vasos a $80 \%$ da capacidade de campo, esta condição foi determinada anteriormente. Ao término do experimento mediuse a altura e coletou-se a parte aérea, que foi seca em estufa $\left(65^{\circ} \mathrm{C}\right)$, até peso constante, para determinação da matéria seca. As folhas foram destacadas dos galhos e moídas para determinação da concentração de macro e micronutrientes, seguindo os procedimentos descritos por Tedesco et al. (1995).

Após a retirada das plantas, o solo dos vasos foi homogeneizado, peneirado, tendo coletada uma amostra de cada unidade experimental para análise

TABELA 1: Descrição dos tratamentos utilizados e teores de nutrientes $\left(\mathrm{g} \mathrm{kg}^{-1}\right)$ nos vermicompostos.

TABLE 1: Treatments description and nutrients concentrations $\left(\mathrm{g} \mathrm{kg}^{-1}\right)$ in the vermicompost.

\begin{tabular}{clcccccc}
\hline & \multicolumn{1}{c}{ Tratamentos } & $\mathrm{C} / \mathrm{N}$ & $\mathrm{N}$ & $\mathrm{P}$ & $\mathrm{K}$ & $\mathrm{Ca}$ & $\mathrm{Mg}$ \\
\hline T1 & Vermicomposto EB & 10,6 & 10,8 & 4,5 & 8,6 & 82,5 & 1,2 \\
T2 & Vermicomposto EO & 10,4 & 15,0 & 2,9 & 20,1 & 137,4 & 4,6 \\
T3 & Vermicomposto LP & 5,9 & 25,1 & 42,0 & 10,2 & 263,9 & 26,0 \\
T4 & Controle (sem adubação) & - & - & - & - & - & - \\
T5 & Controle (adubação mineral NPK) & - & - & - & - & - & - \\
T6 & Vermicomposto da mistura EB + LP (1:1) & 9,5 & 14,5 & 16,6 & 17,7 & 181,4 & 4,6 \\
T7 & Vermicomposto da mistura EO + LP (1:1) & 10,3 & 15,4 & 11,5 & 20,1 & 159,4 & 4,7 \\
T8 & Vermicomposto da mistura EB + RA (1:1) & 10,7 & 16,0 & 22,0 & 28,7 & 280,4 & 19,6 \\
T9 & Vermicomposto da mistura EO + RA (1:1) & 10,1 & 11,2 & 3,1 & 15,6 & 126,4 & 1,9 \\
T10 & Vermicomposto da mistura EB + RF (1:1) & 10,1 & 14,9 & 6,5 & 15,8 & 131,9 & 2,1 \\
T11 & Vermicomposto da mistura EO + RF (1:1) & 9,8 & 15,4 & 3,8 & 18,3 & 88,0 & 1,5 \\
\hline
\end{tabular}

Em que: $\mathrm{EB}=$ esterco bovino; $\mathrm{EO}=$ esterco ovino; $\mathrm{LP}=$ Lodo de parbolização do arroz; RA = resíduos de alimentos; $\mathrm{RF}=$ resíduos de frutas e legumes. (1:1) proporção entre os constituintes (v/v). 
dos teores de $\mathrm{N}$ total, $\mathrm{P}, \mathrm{K}, \mathrm{Ca}, \mathrm{Mg}, \mathrm{Na}, \mathrm{Zn}, \mathrm{Cu}, \mathrm{Fe}$, $\mathrm{Mn}, \mathrm{pH}$ e condutividade elétrica (TEDESCO et al., 1995).

\section{Análise estatística}

O delineamento experimental adotado foi inteiramente casualizado, com três repetições. Os resultados foram submetidos à análise da variância ANOVA, para avaliar o efeito dos tratamentos, e quando significativo, as médias foram comparadas pelo teste Tukey, a 5\% de probabilidade de erro.

\section{RESULTADOS E DISCUSSÃO}

A altura de plantas foi maior com a aplicação do vermicomposto com a mistura de esterco bovino e resíduos alimentos, cujo valor foi $22,6 \%$ superior ao do tratamento controle (Tabela 2). Esse tratamento, entretanto, não diferiu estatisticamente daqueles com aplicação dos vermicompostos com esterco bovino; esterco ovino; tratamento controle com adução mineral; vermicomposto com a mistura de esterco bovino e lodo de arroz parbolizado; mistura de esterco ovino e resíduos de alimentos; e com a mistura de esterco bovino e resíduos de frutas.

A maior produção de matéria seca (MS) da parte aérea das plantas foi obtida com a aplicação de NPK mineral, com valor $166 \%$ superior à MS do tratamento controle negativo (T4) e 75\% superior à média dos tratamentos com aplicação de vermicompostos (Tabela 2). A superioridade do tratamento com adubação mineral pode ser explicada por disponibilidade imediata de nutrientes nos fertilizantes minerais, comparados aos orgânicos, que necessitam primeiro ser mineralizados para liberação de nutrientes. Contudo, esta mineralização mais lenta pode ser favorável à pega da muda e também ao desenvolvimento das plantas após serem transplantadas. Resultados semelhantes são demonstrados por outros autores, em que utilização de vermicomposto foi bem sucedida na produção de mudas de Hovenia dulcis (VOGEL et al., 2001) e Eucaliptus grandis (SCHUMACHER et al., 2001).

A utilização de esterco bovino com vermicomposto de resíduos alimentícios (T8) gerou a mesma produção de matéria seca aérea em relação à adubação com NPK mineral, com produção de $160 \%$ superior ao tratamento controle (T4), evidenciando seu elevado potencial como adubo orgânico. Com exceção dos tratamentos vermicomposto de lodo parbolização arroz (T3) e a mistura de esterco bovino e resíduos de frutas (T11), que foram inferiores na produção de MS da parte aérea e semelhantes ao tratamento controle. Em média, os demais tratamentos com vermicomposto aumentaram a MS da acácia-negra em 68\%. Apesar da baixa correlação observada entre a matéria seca e a altura das plantas $(\mathrm{r}=0,46)$, constata-se que os tratamentos T3 e T11 também foram os que apresentaram os menores valores para altura de plantas, embora estes não tenham diferenciado significativamente dos demais tratamentos com adubação orgânica. Uma avaliação conjunta dos dados de altura de plantas e matéria seca evidenciou que os tratamentos com esterco bovino e resíduos de alimentos e adubação com NPK mineral foram os melhores para o crescimento plantas, com efeitos semelhantes.

Outros trabalhos demonstram que a aplicação de vermicomposto em mudas melhorou o crescimento vegetal (VOGEL et al., 2001; SCHUMACHER et al., 2001; CORREIA et al., 2005; SILVA et al., 2008; BALDOTTO et al., 2012); já no caso de mudas de gravioleira, o vermicomposto também estimulou a parte biológica do solo, como o aumento da formação de arbúsculos dos fungos micorrízicos no córtex das plantas, proporcionando melhor condição de crescimento vegetal e atividade microbiana no solo (SILVA et al., 2008). Além disso, com a aplicação de vermicomposto em plantas de milho foi observado melhora no efeito sinérgico quando combinada com a aplicação de bactérias endofíticas, melhorando as condições de crescimento das plantas e, consequentemente, promovendo o crescimento inicial do milho (BALDOTTO et al., 2012).

Embora a adição de fertilizantes convencionais contribua para que a mineralização dos nutrientes seja mais fácil e rápida, a adubação orgânica promove as condições ambientais do solo, melhorando as condições bióticas e podendo, em médio prazo, promover crescimento das plantas. Nesta linha, Pandolfo et al. (2003) relatam que a adubação orgânica em cultivos de Ilex paraguariensis apresentou resposta significativa à produção de massa verde e atribuíram não somente aos teores de minerais contidos no adubo, mas a uma combinação de efeitos estimulados pela presença de matéria orgânica e minerais, como aumento da atividade microbiana. Além disso, plantas com crescimento mais longo, como no caso de plantas arbóreas, é favorável uma taxa de mineralização mais lenta para aumentar a disponibilidade de 
nutrientes durante o tempo de desenvolvimento.

As maiores concentrações de macronutrientes no tecido foliar das plantas foi observada nos tratamentos com aplicação do vermicomposto originado do lodo de parbolização de arroz (T3) ou em sua mistura com esterco bovino (T6) e esterco ovino (T7) (Tabela 2). Além disso, as maiores concentrações de $\mathrm{P}$ e de $\mathrm{Mg}$ nas plantas com aplicação do vermicomposto de lodo de parbolização do arroz (T3) podem estar refletindo a elevada concentração desses dois nutrientes nesse material (Tabela 1). Por outro lado, os menores teores observados no tratamento NPK mineral (T5) podem ser decorrentes de um efeito de diluição na concentração foliar, já que este tratamento apresentou maior produção de MS da parte aérea das plantas (Tabela 2). Para os elementos $\mathrm{N}$ e P, destaca-se o tratamento T3, sendo que para $\mathrm{P}$, o tratamento $\mathrm{T} 3$ não diferiu do T6 e T7.
Os tratamentos T6 e T7 não diferem entre si para os elementos $\mathrm{K}$ e $\mathrm{Ca}$ e o tratamento $\mathrm{T} 6$ não diferiu do tratamento T3. Isto pode ser devido à composição química dos materiais, além disso, resíduos de estercos, tanto bovinos quanto ovinos, têm uma taxa transformação diferente de resíduos de origem dos alimentos e frutas, formando um vermicomposto com características diferentes.

No geral, foi observado que a adição dos tratamentos T3, T6 e T7 promoveram melhores resultados na concentração de nutrientes na parte aérea de mudas de acácia-negra (Tabela 2). A concentração de nutrientes pode ser um indicativo de nutrição devido ao diferente tipo de fonte de nutriente aplicado. A maior ou menor eficiência de uso de nutrientes das diferentes espécies arbóreas pode ser o reflexo de uma adaptação às perdas anuais e pode aumentar sua reutilização (SCHUMACHER, 1995). Caldeira et al. (2002) constataram uma variação na

TABELA 2: Altura de plantas, matéria seca (MS) da parte aérea e concentração de macro e micronutrientes nas folhas da acácia-negra após 180 dias de cultivo.

TABLE 2: Plants height, dry mass (MS) of the shoots and macro and micronutrient concentration in shoots of the Acacia mearnsii, after 180 days of cultivation.

\begin{tabular}{|c|c|c|c|c|c|c|c|c|c|c|c|}
\hline Trat. & Altura & MS & $\mathrm{N}$ & $\mathrm{P}$ & $\mathrm{K}$ & $\mathrm{Ca}$ & $\mathrm{Mg}$ & $\mathrm{Fe}$ & $\mathrm{Mn}$ & $\mathrm{Zn}$ & $\mathrm{Cu}$ \\
\hline & $\mathrm{cm}$ & --g -- & \multicolumn{5}{|c|}{ - } & \multicolumn{4}{|c|}{-------------- $\mathrm{mg} \mathrm{kg}^{-1}$--------- } \\
\hline $\mathrm{T} 1$ & $79 a b$ & $\begin{array}{c}19,51 \\
\text { abc }\end{array}$ & $9,73 \mathrm{ab}$ & $0,97 \mathrm{c}$ & $6,41 \mathrm{a}$ & $13,48 \mathrm{~b}$ & $4,57 \mathrm{e}$ & $177,8 \mathrm{a}$ & 96,3 a & $25,1 \mathrm{~b}$ & $7,0 \mathrm{~b}$ \\
\hline $\mathrm{T} 2$ & $75 \mathrm{ab}$ & $\begin{array}{c}17,16 \\
a b c\end{array}$ & $8,65 \mathrm{~b}$ & $5,06 \mathrm{bc}$ & $11,88 \mathrm{a}$ & $13,59 \mathrm{~b}$ & 5,37 cde & $165,2 \mathrm{a}$ & 82,4 a & $56,5 \mathrm{ab}$ & $\begin{array}{c}31,4 \\
a b\end{array}$ \\
\hline $\mathrm{T} 3$ & $70 \mathrm{~b}$ & $9,02 \mathrm{c}$ & $12,44 \mathrm{a}$ & $11,83 \mathrm{a}$ & $10,82 \mathrm{a}$ & $15,80 \mathrm{ab}$ & $7,75 \mathrm{ab}$ & $102,2 \mathrm{a}$ & $109,7 \mathrm{a}$ & 64,3 a & $79,5 \mathrm{a}$ \\
\hline $\mathrm{T} 4$ & $73 \mathrm{~b}$ & $\begin{array}{c}10,54 \\
\mathrm{dc}\end{array}$ & $8,09 \mathrm{~b}$ & $1,13 \mathrm{c}$ & $7,80 \mathrm{a}$ & $16,09 \mathrm{ab}$ & $4,93 \mathrm{de}$ & $162,4 \mathrm{a}$ & $102,8 \mathrm{a}$ & $29,4 \mathrm{~b}$ & $\begin{array}{c}16,6 \\
a b\end{array}$ \\
\hline T5 & $74 \mathrm{ab}$ & $28,06 \mathrm{a}$ & $9,22 \mathrm{~b}$ & $2,26 \mathrm{bc}$ & 7,06 a & $12,55 \mathrm{~b}$ & $3,93 \mathrm{e}$ & $126,0 \mathrm{a}$ & $158,9 \mathrm{a}$ & $27,8 \mathrm{~b}$ & $\begin{array}{c}10,5 \\
a b\end{array}$ \\
\hline $\mathrm{T} 6$ & $82 \mathrm{ab}$ & $\begin{array}{c}14,74 \\
\text { bc }\end{array}$ & $9,56 \mathrm{ab}$ & 11,11 a & $13,79 \mathrm{a}$ & $22,92 \mathrm{a}$ & $8,74 \mathrm{a}$ & $147,0 \mathrm{a}$ & $102,3 \mathrm{a}$ & 64,9 a & $\begin{array}{c}29,7 \\
a b\end{array}$ \\
\hline $\mathrm{T} 7$ & $73 b$ & $\begin{array}{c}16,79 \\
\text { bc }\end{array}$ & $9,16 \mathrm{~b}$ & $7,21 \mathrm{ab}$ & $13,70 \mathrm{a}$ & $17,99 \mathrm{ab}$ & $7,22 \mathrm{abc}$ & $127,4 \mathrm{a}$ & 97,3 a & $51,4 \mathrm{ab}$ & $\begin{array}{c}15,7 \\
a b\end{array}$ \\
\hline $\mathrm{T} 8$ & $94 \mathrm{a}$ & $\begin{array}{c}23,54 \\
\mathrm{ab}\end{array}$ & $8,93 \mathrm{~b}$ & $5,57 \mathrm{bc}$ & $12,26 \mathrm{a}$ & $14,59 \mathrm{ab}$ & 5,62 bcde & $109,2 \mathrm{a}$ & 87,9 a & $36,5 \mathrm{ab}$ & $\begin{array}{c}11,3 \\
a b\end{array}$ \\
\hline T9 & $78 a b$ & $\begin{array}{c}15,56 \\
\text { bc }\end{array}$ & $9,16 \mathrm{~b}$ & $3,92 \mathrm{bc}$ & $10,86 \mathrm{a}$ & $16,20 \mathrm{ab}$ & 5,65 bcde & $113,4 \mathrm{a}$ & 98,3 a & $38,5 \mathrm{ab}$ & $\begin{array}{c}20,9 \\
a b\end{array}$ \\
\hline $\mathrm{T} 10$ & $79 a b$ & $\begin{array}{c}16,05 \\
b c\end{array}$ & $8,14 \mathrm{~b}$ & $3,71 \mathrm{bc}$ & $9,15 \mathrm{a}$ & $15,30 \mathrm{ab}$ & 6,00 bcde & $142,8 \mathrm{a}$ & 89,4 a & $35,1 \mathrm{ab}$ & $\begin{array}{c}19,2 \\
a b\end{array}$ \\
\hline $\mathrm{T} 11$ & $66 \mathrm{~b}$ & $12,10 \mathrm{c}$ & $9,50 \mathrm{ab}$ & $3,28 \mathrm{bc}$ & 9,61 a & $15,34 \mathrm{ab}$ & $6,89 \mathrm{abcd}$ & $105,0 \mathrm{a}$ & $101,8 \mathrm{a}$ & $39,8 \mathrm{ab}$ & $\begin{array}{c}27,1 \\
\mathrm{ab}\end{array}$ \\
\hline
\end{tabular}

Em que: Médias seguidas de mesma letra não diferem entre si pelo teste Tukey, a 5\%. Tratamentos: T1) vermicomposto de esterco bovino (EB); T2) vermicomposto de esterco ovino (EO); T3) vermicomposto de lodo de parbolização arroz (LP); T4) tratamento controle (sem adubação); T5) tratamento controle com adubação mineral; T6) mistura de EB e LP; T7) mistura de EO e LP; T8) mistura de EB e vermicomposto de resíduos de alimentos (RA); T9) mistura de EO e RA; T10) mistura de EB e vermicomposto de resíduos de frutas (RF); T11) mistura de EO e RF. 
eficiência nutricional com macronutrientes entre diferentes tipos de Acacia mearnsii.

Com exceção do $\mathrm{N}$, os teores dos demais macronutrientes são maiores que os observados por Caldeira et al. (2000) em folhas da biomassa aérea de plantas de Acacia mearnsii, com idade de 2,4 anos. Entretanto, se comparados às concentrações na parte aérea de mudas de Acacia mangium (com 90 dias) obtidas em diferentes substratos, com adubação orgânica e mineral (CUNHA et al., 2006), os teores de macronutrientes deste trabalho, de um modo geral, foram semelhantes ou menores. Considerando as diferenças entre espécies e idades das plantas das referências citadas e do presente trabalho (com 180 dias) podem-se admitir para os mesmos, concentrações diferenciadas. $\mathrm{O}$ fato de a idade e a espécie influenciarem na concentração de nutrientes nas espécies arbóreas, de certo modo, dificulta a comparação de resultados entre diferentes trabalhos.

As variações na concentração do $\mathrm{Fe}$ e do Mn no tecido foliar, nos diferentes tratamentos não foram estatisticamente significativas (Tabela 2). Com relação ao $\mathrm{Zn}$, as maiores concentrações no tecido vegetal foram obtidas nos tratamentos T3 e T6, os quais superaram as concentrações obtidas nos tratamentos controle (T4), T1, T5, não diferindo dos demais tratamentos, esta maior concentração pode ser advinda de disponibilização deste nutriente, pois estes tratamentos são compostos com os lodos biológicos de parbolização de arroz, na qual o Zn é encontrado em maior quantidade. As concentrações de $\mathrm{Fe}$ e $\mathrm{Zn}$ deste trabalho estão dentro do intervalo observado por Caldeira et al. (2002), para a mesma espécie, mas em outra situação de estudo, enquanto que as concentrações de $\mathrm{Mn}$ e $\mathrm{Cu}$ foram superiores.

$\mathrm{O} \mathrm{pH}$ do solo em todos os tratamentos manteve-se entre 5,4 e 5,8, após os 180 dias de cultivo em casa de vegetação, a exceção do tratamento com adubação mineral (Tabela 3). Isto pode ser devido à decorrência da nitrificação do nitrogênio amoniacal, que aumenta a produção de íons $\mathrm{H}^{+}$, diminuindo o $\mathrm{pH}$ do solo (MEURER, 2004). Em relação à condutividade elétrica, os maiores valores obtidos nos tratamentos mistura de esterco bovino e lodo de parbolização (T6), esterco bovino e de resíduos alimentos (T8) e esterco ovino e esterco de alimentos (T9). Isto pode ser devido aos elevados teores de $\mathrm{Ca}$ e $\mathrm{Mg}$ obtidos nos mesmos tratamentos.

O tratamento T11 promoveu os menores teores de $\mathrm{Ca}$ e $\mathrm{Mg}$ no solo (Tabela 3). O que pode ser devido às baixas concentrações encontradas destes nutrientes na mistura do vermicomposto em questão (Tabela 1), enquanto que, no tratamento $\mathrm{T} 8$, estes elementos se destacaram perante os outros tratamentos, por apresentarem os teores mais elevados no solo. O maior valor de $\mathrm{K}$ no solo decorreu da aplicação do tratamento T2 (Tabela 3).

A adição dos vermicompostos ao solo em comparação ao tratamento controle (T5) aumentou sua fertilidade, mesmo após o cultivo, especialmente com relação ao fósforo, potássio e magnésio. Segundo o Manual de Adubação e Calagem do RS/SC (CQFS, 2004), o fósforo, que no tratamento controle apresentou teor baixo, elevouse para classe alta no tratamento com mistura de esterco bovino e de resíduos de frutas (T10) e para muito alto nos demais tratamentos. O potássio, cujo teor na testemunha apresentava-se médio, resultou em nível alto nos demais tratamentos praticamente duplicando seus teores. O magnésio com teor baixo na testemunha passou a médio, na maior parte dos tratamentos.

A adubação orgânica aumentou os teores de carbono no solo em comparação ao tratamento controle (T4) e à adubação mineral (T5) (Tabela 4). O maior conteúdo de C orgânico foi obtido no tratamento T8, fato explicado por este vermicomposto ser o que apresenta maior quantidade de $\mathrm{C}$ total. Utilizando-se do fator de conversão do carbono para a matéria orgânica de 1,73, pode-se constatar que, com exceção do tratamento T11, o incremento de matéria orgânica dos demais tratamentos com vermicompostos foram suficientes para elevar os seus teores do nível baixo para o médio (CQFS, 2004).

A fertilização das mudas de acácia-negra com adubação mineral adequada estipulada pelo Manual de Adubação de Solos (CQFS, 2004) mostrou-se o melhor tratamento na produção das mudas de acácia-negra. Contudo, o tratamento com a utilização do vermicomposto da mistura de esterco bovino e de resíduos de alimentos obteve resultados semelhantes com a adubação mineral. Devido aos efeitos benéficos da adição de resíduos orgânicos como a melhoria da atividade biológica, condicionamento de solo, estabilidade física, menor taxa de mineralização, entre outros (MEURER, 2004; MOREIRA e SIQUEIRA, 2006), a adução orgânica mostra-se como uma adubação mais interessante ao ambiente solo, e meio ambiente. A reutilização de resíduos tem um aspecto ambientalmente correto, sendo retirado do ambiente um resíduo, que, se 
TABELA 3: Atributos do solo, após180 dias de cultivo de acácia-negra com os diferentes tratamentos. TABLE 3: Soil attributes after 180 days of growth of Acacia mearnsii with different treatments.

\begin{tabular}{|c|c|c|c|c|c|c|c|}
\hline \multirow{2}{*}{ Tratamento } & \multirow{2}{*}{$\mathrm{pH}_{\mathrm{H} 2 \mathrm{O}}$} & $\mathrm{CE}$ & $\mathrm{P}$ & $\mathrm{K}$ & $\mathrm{Ca}$ & $\mathrm{Mg}$ & \multirow{2}{*}{$\begin{array}{c}\text { Corg } \\
\%\end{array}$} \\
\hline & & $\mu \mathrm{S} \mathrm{cm}^{-1}$ & \multicolumn{2}{|c|}{-------mg kg-1------- } & \multicolumn{2}{|c|}{------cmol $\mathrm{dm}^{-3}$----- } & \\
\hline $\mathrm{T} 1$ & $5,7 \mathrm{ab}$ & $161 \mathrm{~d}$ & $25,7 \mathrm{e}$ & $95,6 \mathrm{~b}$ & $0,86 \mathrm{bc}$ & 0,49 bcd & $1,49 \mathrm{~d}$ \\
\hline $\mathrm{T} 2$ & $5,8 \mathrm{a}$ & $185 \mathrm{~b}$ & 24,5 ef & $110,6 \mathrm{a}$ & $0,82 \mathrm{bc}$ & $0,57 \mathrm{abc}$ & $1,50 \mathrm{~d}$ \\
\hline T3 & $5,6 \mathrm{bc}$ & $178 \mathrm{bc}$ & $84,09 a$ & $58,4 \mathrm{~cd}$ & $0,888 \mathrm{c}$ & $0,56 \mathrm{bc}$ & $1,36 \mathrm{de}$ \\
\hline $\mathrm{T} 4$ & $5,4 \mathrm{~d}$ & $118 \mathrm{e}$ & $4,14 \mathrm{~g}$ & $48,4 \mathrm{~d}$ & $0,64 \mathrm{~d}$ & $0,32 \mathrm{e}$ & 1,24 ef \\
\hline T5 & $5,0 \mathrm{e}$ & $171 \mathrm{bcd}$ & $51,13 \mathrm{c}$ & $61,6 \mathrm{c}$ & $0,76 \mathrm{~cd}$ & $0,33 \mathrm{e}$ & $1,19 \mathrm{f}$ \\
\hline T6 & $5,7 \mathrm{ab}$ & $212 \mathrm{a}$ & $64,41 b$ & $102,1 \mathrm{ab}$ & $0,97 \mathrm{abc}$ & $0,61 \mathrm{a}$ & $1,51 \mathrm{~cd}$ \\
\hline $\mathrm{T} 7$ & $5,6 \mathrm{bc}$ & $183 \mathrm{~b}$ & $38,31 \mathrm{~d}$ & $100,4 \mathrm{ab}$ & $0,88 \mathrm{bc}$ & $0,58 \mathrm{ab}$ & $1,51 \mathrm{~cd}$ \\
\hline $\mathrm{T} 8$ & $5,5 \mathrm{~cd}$ & $213 \mathrm{a}$ & $59,87 b$ & $96,2 \mathrm{~b}$ & $1,1 \mathrm{a}$ & $0,61 \mathrm{a}$ & $2,02 \mathrm{a}$ \\
\hline T9 & $5,4 \mathrm{~cd}$ & $203 \mathrm{a}$ & $20,82 \mathrm{ef}$ & $96,6 \mathrm{~b}$ & $0,95 \mathrm{ab}$ & $0,54 \mathrm{abc}$ & $1,68 \mathrm{~b}$ \\
\hline $\mathrm{T} 10$ & $5,4 \mathrm{~cd}$ & $165 \mathrm{~cd}$ & $20,82 \mathrm{ef}$ & $96,6 \mathrm{~b}$ & $0,84 \mathrm{bc}$ & $0,47 \mathrm{~cd}$ & $1,66 \mathrm{bc}$ \\
\hline $\mathrm{T} 11$ & $5,5 \mathrm{~cd}$ & $160 \mathrm{~d}$ & $18,28 \mathrm{f}$ & $97,5 \mathrm{~b}$ & $0,72 \mathrm{~cd}$ & $0,42 \mathrm{de}$ & $1,30 \mathrm{ef}$ \\
\hline
\end{tabular}

Em que: Médias seguidas de mesma letra minúscula na coluna, não diferem entre si pelo teste de Tukey a $5 \%$. Tratamentos: T1) vermicomposto de esterco bovino (EB); T2) vermicomposto de esterco ovino (EO); T3) vermicomposto de lodo de parbolização arroz (LP); T4) tratamento controle (sem adubação); T5) tratamento controle com adubação mineral; T6) mistura de EB e LP; T7) mistura de EO e LP; T8) mistura de EB e vermicomposto de resíduos de alimentos (RA); T9) mistura de EO e RA; T10) mistura de EB e vermicomposto de resíduos de frutas (RF); T11) mistura de EO e RF.

descartado inadequadamente, torna-se um problema ambiental. No caso dos resíduos de alimentos, estercos de animais, e lodo de parbolização de arroz tornam-se potenciais fertilizantes com teores adequados de nutrientes. Assim, só o fato da retirada de um resíduo poluente do ambiente e a transformação deste em um insumo na produção de mudas de acácia-negra seria uma solução adequada. Além disso, outros vermicompostos produzidos com os resíduos de esterco bovino, esterco ovino e resíduos de frutas foram benéficos à produção de mudas de acácia-negra.

\section{CONCLUSÕES}

A adição dos vermicompostos dos resíduos de esterco bovino (T1), bem como a mistura de vermicomposto de esterco bovino e resíduos de alimentos (T8), favoreceram o incremento de matéria seca da acácia-negra.

$\mathrm{O}$ vermicomposto de lodo de parbolização arroz (T3) elevou as concentrações de P, N, K, Zn e $\mathrm{Cu}$, enquanto que e a mistura de vermicomposto de esterco bovino e lodo de parbolização arroz (T6) elevou as concentrações de $\mathrm{P}, \mathrm{K}$ e $\mathrm{Zn}$ nas folhas de acácia-negra.

A adição de vermicompostos ao solo aumentou a disponibilidade de $\mathrm{P}, \mathrm{K}$ e $\mathrm{Mg}$ para as plantas.
A utilização de vermicomposto dos tratamentos T8, T1 e T3 demonstrou potencial na produção de mudas de acácia-negra, sendo uma possibilidade na suplementação e/ou substituição da adubação convencional.

\section{REFERENCIAS BIBLIOGRÁFICAS}

ANTONIOLLI, Z.I. et al. Minhocultura e vermicompostagem. Santa Maria: Editora da Universidade Federal de Santa Maria, 2002 (Informe Técnico). 24p.

ANTUNES, R.M. Humificação de resíduos orgânicos durante a vermicompostagem e seu efeito nos atributos químicos do solo e no crescimento inicial de acácia negra (Acacia mearnsii). 2009. 86f. Tese (Doutorado em Ciência do Solo). Programa de Pós-Graduação em Agronomia. Faculdade de Agronomia Eliseu Maciel. Universidade Federal de Pelotas, Pelotas, 2009.

BALDOTTO, L.E.B. et al. Growth promotion of pineapple 'vitória' by humic acids and Burkholderiaspp. during acclimatization. Revista Brasileira de Ciência do Solo, Viçosa, v. 34, p.1593-1600, 2010.

BALDOTTO, L.E.B. et al.Initial growth of maize in response to application of rock phosphate, vermicompost and endophyticbactéria. Revista 
Ceres, Viçosa, v. 59, n.2, p.262-270, 2012.

CALDEIRA, M.V.W. et al. Concentração e redistribuição de nutrientes nas folhas e no folhedo em um povoamento de Acacia mearnsii De Wild. No Rio Grande do Sul. Ciência Florestal, Santa Maria, v. 9, n.1. p.19-24. 1999.

CALDEIRA, M.V.W.et al. Ciclagem de nutrientes em Acácia mearnsiide Wild.v. quantificação do conteúdo de nutrientes na biomassa aérea de Acacia mearnsii De Wild. Procedência australiana. Ciência Rural, Santa Maria, v. 30.n.6, p.977-982. 2000.

CALDEIRA, M.V.W., NETO, R.M.R.. SCHUMACHER, M.V. Acúmulo de micronutrientes em um povoamento de acácia-negra (Acacia mearnsii De Wild.) Procedência Bodalla-Austrália. Floresta, Curitiba, v. 33, n.1, p.73-78. 2002.

CALDEIRA, M.V.W.; NETO, R.M.R.; SCHUMACHER, M.V. Eficiência do uso de micronutrientes e sódio em três procedências de acácia-negra (Acacia mearnsii De Wild.). Revista Árvore, Viçosa, v. 28, n. 1, p.39-47, 2004.

COMISSÃO DE QUÍMICA E FERTILIDADE DO SOLO. Manual de adubação e de calagem para os estados do Rio Grande do Sul e de Santa Catarina. 2004. Porto Alegre, 394 p.

CORREIA, D. et al. Efeito de substratos na formação de porta-enxertos de Psidium guajava L. cv. ogawa em tubetes. Revista Brasileira de Fruticultura, Jaboticabal, v. 27, n.1, p.88-91, 2005.

CUNHA, A.M. et al. 2006 Efeito de diferentes substratos sobre o desenvolvimento de mudas de Acacia sp. Revista Árvore, Viçosa, v.30, n.2, p. 207-214.

DANNER, M.A. et al. Formação de mudas de jabuticabeira (Plinia sp.) em diferentes substratos e tamanhos de recipientes. RevistaBrasileira de Fruticultura, Jaboticabal, v. 29, n.1, p.179-182, 2007.

HASHEMIMAJD, K.; JAMAATI-SOMARIN, S. Contribution of organic bulking materials on chemical quality of sewage sludge vermicompost. Ciência e Agrotecnologia, Lavras, v. 35, n.6, p.1077-1084, 2011.

HIGA, A.R.; RESENDE, M.D.V. Breeding Acacia mearnsii in Southern Brazil. In: Australian tree species research in China, Zhabgzhou, 1992. Proceedings, 48..., Canberra: ACIAR, 1994. p. 158-160.

KLEINPAUL, I.S. et al. Plantio misto de Eucalyptus urograndis e Acacia mearnsii em sistema agroflorestal: I - produção de biomassa. Ciência
Florestal, Santa Maria, v. 20, n. 4, p. 621-627, out.-dez., 2010.

KIEHL, S.S. et al. Efeito de diferentes níveis de calcário, adubação fosfatada e potássica no desenvolvimento aéreo e radicular da acácia negra (Acacia mearnsii De Wild). Anais... XXIII Reunião Brasileira de Fertilidade do Solo e Nutrição de Plantas, Caxambu, MG. 1998.1 CD.

MARTINS, C.C. et al.Efeito do sombreamento e do substrato sobre a germinação e o crescimento de plântulas de Acacia mangium e Acacia mearnsii. Ciência Florestal, Santa Maria, v. 22, n.2, p.283-293, 2012.

MEURER, E.J. Fundamentos de Química do Solo. Ed. Gênesis. Porto Alegre. 2a Edição. 2004. 290p. MOREIRA, F.M.S.;SIQUEIRA, J.O. Microbiologia e bioquímica do solo. Lavras: UFLA, 2006. 729p. NEVES, C.S.V.J. et al. Efeitos de substratos e recipientes utilizados na produção das mudas sobre a arquitetura do sistema radicular de árvores de acácia-negra. Revista Árvore, Viçosa, v. 29, n.6, p.897-905, 2005.

PANDOLFO, C.M. et al. Resposta da erva-mate (Ilex paraguariensis St. Hil.) à adubação mineral e orgânica em um Latossolo Vermelho alumino férrico. Ciência Florestal, Santa Maria, v. 13, n. 2, p. 37-45, 2003.

PELIZZA, T.R. et al. Microestaquia em mirtileiro com diferentes porções do ramo e substratos. Bragantia, Campinas, v. 70, n.2, p.319-324, 2011. RODDA, M.R.C. et al. Estímulo no crescimento e na hidrólise de ATP em raízes de alface tratadas com humatos de vermicomposto. II - efeito da fonte de vermicomposto. Revista Brasileira de Ciência do Solo, Viçosa, v. 30, p.657-664, 2006.

SCHUMACHER, $M$. et al. Influência do vermicomposto na produção de mudas de Eucalyptus grandis Hill ex Maiden. Ciência Florestal, Santa Maria, v. 11, n.2, p. 121-130, 2001.

SILVA; P.R.D. et al. Estudo preliminar do vermicomposto produzido a partir de lodo de esgoto doméstico e solo.Eclética Química, São Paulo, v. 35, n.3, p.61-67, 2010.

SILVA, D.A.A. et al. Uso de vermicomposto favorece o crescimento de mudas de gravioleira (Annona muricata L. 'Morada') associadas a fungos micorrízicos arbusculares. Acta Botânica Brasiliensis, São Paulo, v. 22, n.3, p.863-869, 2008. SORRENTI, G.B. et al. Influência da adubação orgânica no crescimento de tangerineira cv Clemenules e nos atributos químicos e microbiológicos do solo. Revista Brasileira de 
Fruticultura, Jaboticabal, v. 30, n.4, p.1129-1135, 2008.

SCHUMACHER, M.V. et al. Influência do vermicomposto na produção de mudas de Eucalyptus grandis Hill ex Maiden. Ciência Florestal, Santa Maria, v.11, n.2, p.121-130, 2001. STRECK, E.V. et al. Solos do Rio Grande do Sul. Porto Alegre, EMATER-RS / UFRGS, 2002. 126 p. TEDESCO, J. M. et al. Análise de solo, planta e outros materiais. $2^{\mathrm{a}}$ ed. Porto Alegre. UFRGS. 1995.

TEDESCO, M.J. et al. Resíduos orgânicos no solo e impactos no ambiente. In: SANTOS, G.A.; CAMARGO, F.A.O. Fundamentos da matéria orgânica do solo, ecossistemas Tropicais e Subtropicais. Porto Alegre: Editora Gênesis, 1999. p.159-192.

TIBAU, A.O. Matéria orgânica do solo. In: Matéria orgânica e fertilidade do solo. São Paulo: Ed.
Nobel, 1984. p.49-182.

TONETTO, L.; STEIN, P.P. Silvicultura da acácia negra (Acacia mearnsii De Wild.) no Brasil. Florestar Estatístico, v.4, n.12, p. 11-16, 1997.

VARGAS, L.K. et al. Diversidade genética e eficiência simbiótica de rizóbios noduladores de acácia-negra de solos do Rio Grande do Sul. Revista Brasileira de Ciência do Solo, Viçosa, v. 31, p.647-654, 2007.

VIERA, M.; SCHUMACHER,M.V.; LIBERALESSO, E. Crescimento e produtividade de povoamentos mono específicos e mistos de eucalipto e acácia-negra. Pesquisa Agropecuária Tropical, Goiânia, v. 41, n.3, p.415-421, 2011.

VOGEL, H.L.M. et al. Utilização de vermicomposto no crescimento de mudas de Hovenia dulcis Thunberg. Ciência Florestal, Santa Maria, v. 11, n.1, p.21-27, 2001. 